\title{
Retraction Note to: Mobile network intrusion detection for loT system based on transfer learning algorithm
}

\author{
Lianbing Deng ${ }^{1,2} \cdot$ Daming $\mathrm{Li}^{3,4,5} \cdot$ Xiang $\mathrm{Yao}^{2} \cdot$ Haoxiang Wang ${ }^{6,7}$
}

Published online: 3 February 2021

(C) Springer Science+Business Media, LLC, part of Springer Nature 2021

\section{Retraction to: \\ Cluster Computing (2019) 22:S9889-S9904 https://doi.org/10.1007/s10586-018-1847-2}

The Editors-in-Chief have retracted this article.

After publication, it has come to the Editors' attention that the email address provided for Dr. David Cox as part of the submission did not belong to him. The corresponding author and Dr. Cox have confirmed that Dr. Cox did not contribute to the article. Dr David Cox's name has been removed from the author list and his biographical information included in the article pdf has been redacted. Moreover, post-publication peer review found that the methods and results are inadequately described to support the conclusions presented in the article. The Editors-inChief therefore no longer have confidence in the reliability of the work presented.

Publisher's Note Springer Nature remains neutral with regard to jurisdictional claims in published maps and institutional affiliations.

The original article can be found online at https:// doi.org/10.1007/s10586-018-1847-2.

\footnotetext{
Daming Li

lidaming@yahoo.com

1 Huazhong University of Science and Technology, Wuhan, China

2 Zhuhai Da Hengqin Science and Technology Development Co., Ltd., Hengqin, China

3 The Post-Doctoral Research Center of Zhuhai Da Hengqin Science and Technology Development Co., Ltd., Hengqin, China

4 City University of Macau, Taipa, Macau

5 International Postdoctoral Science and Technology Research Institute Co., Ltd, Wuhan, China

6 Cornell University, Ithaca, NY, USA

7 GoPerception Laboratory, New York, NY, USA
} 